\title{
Rsa Algorithm WiTH A NeW APPROACH ENCRYPTION AND DECRYPTION MESSAGE TEXT BY ASCII
}

\author{
Ahmad Steef $^{1}$, M. N. Shamma ${ }^{2}$ and A. Alkhatib ${ }^{3}$ \\ ${ }^{1}$ Department of Mathematics, Al Baath University- Homs- Syria. \\ ${ }^{2}$ Basic Sciences department- the Mechanical and Electrical Engineering- Damascus \\ University- Syria. \\ ${ }^{3}$ Department of mathematics \& Faculty sciences - Al Baath University- Homs- Syria.
}

\begin{abstract}
In many research works, there has been an orientation to studying and developing many of the applications of public-key cryptography to secure the data while transmitting in the systems, In this paper we present an approach to encrypt and decrypt the message text according to the ASCII(American Standard Code for Information Interchange) and RSA algorithm by converting the message text into binary representation and dividing this representation to bytes( $8 s$ of $0 s$ and $1 s)$ and applying a bijective function between the group of those bytes and the group of characters of ASCII and then using this mechanism to be compatible with using RSA algorithm, finally, Java application was built to apply this approach directly.
\end{abstract}

\section{KEYWORDS}

Cryptography, RSA Algorithm, ASCII(American Standard Code for Information Interchange), Binary Number Systems, Java Language.

\section{INTRODUCTION}

Public-Key Cryptography[1 ][2] is the most common major factor for security and protection of Systems. It is a strong technique to secure the data transmitting within Systems. Public-Key Cryptography uses two roles(encrypting and decrypting functions). Encrypting function encrypts massage and converts it to cipher(gibberish) "C", Decrypting Function is applied to "C" to coming back to original message "M". decryption and encryption functions are inverse each other and use distinct keys(numbers)[1]. In a special case, the RSA Algorithm[1 ][2], the most strong common applications of Public-Key Cryptography which published by Rivest, Shamir and Adelman 1978. It depends on the difficult problem in mathematics which is "factorization problem". This Algorithm was built depending on exponential function E(Encryption Function)has inverse $\mathrm{D}$ (Decryption Function), but it is difficult to discovering it, because this depends on the " $\mathrm{P}=\mathrm{NP}$ Problem[2]" is one of the biggest open problems in both mathematics and computer science. RSA Algorithm is used to encrypt and decrypt text according to represent alphabets $(\mathrm{A}, \mathrm{B}, \ldots \ldots \mathrm{Z})$ as decimal numbers(from 0 ..to 25)[ 2], but what about if the text message includes numbers and some characters like: \&@!*...? what about encryption and decryption text message by RSA according to ASCII(American Standard Code for Information Interchange)[3]. This paper focused on cryptography to secure the message while transmitting in the systems, so, protecting 
confidentiality and integrity of information. In this paper we will illustrate the use of RSA to encrypt and decrypt message which is to be transmitted from sender to receiver by a new approach related of ASCII. It's easy to convert any character in ASCII into associated number but How can we convert big numbers to the associated text by ASCII?. The answer of all inquiries was included in this paper, and a numerical example applied to illustrating the basic idea in applicable way, finally, Java application was built to apply this approach directly.

\section{RSA ALgORITHM.}

RSA Algorithm[1][2] is the most strong common applications of Public-Key Cryptography which published by Rivest, Shamir and Adelman 1978. It uses two distinct keys(two numbers), publickey which possible to be known to every one and the other is private-key which is secured and not allowed to exchange between the sender and reciver. This algorithm described as following:

1- Choose two distinct large random prime numbers $\mathrm{p}$ and $\mathrm{q}$.

2- Compute $n=p * q$,

3- Compute Euler's function of $\mathrm{n}: \emptyset(n)=(p-1) *(q-1)$.

4- Choose an integer $e$ such that $1<e<\emptyset(n)$ and $\operatorname{gcd}(e, \emptyset(n))=1$,

5- Compute $d$ such that: e.d $=1(\bmod \emptyset(n))$.

The number $e$ is the public - key and $d$ is the private -key. Let $\mathrm{M}$ is a message need to be encrypted and get cipher $\mathrm{C}$, so, in the first the message $\mathrm{M}$ represented as blocks of numbers every value of block must be less than the number "n" [2]. For any block number $M_{i} ; i \in I$, such that: $M_{i} \in Z_{n}=\{0,1,2,3, \ldots \ldots n-1\}$, the Encryption role of this algorithm is $: C_{i} \equiv M_{i}{ }^{e}(\bmod n)$. and, the Decryption role is $: M_{i} \equiv C_{i}^{d}(\bmod n)$.

Note: $\mathrm{M}$ divided into blocks as form: $\left(M_{1}\left|M_{2}\right| \ldots \ldots M_{i} \mid \ldots ..\right)$ and Cipher $\mathrm{C}$ will be as form: $\left(C_{1}\left|C_{2}\right| \ldots \ldots, C_{i} \mid \ldots ..\right)$.

\section{AdDRESSEd Problem}

Suppose we have message text and want to encrypt it by RSA algorithm depending on ASCII, first, we will represent it as numbers by convert every character of the message text into associated number depending on ASCII, then we will apply RSA algorithm on those numbers which presented in, but when we want to coming back to original message, there will be problem because there are characters in ASCII represented as decimal numbers of two digits and the others represented as numbers in a three digits, for example the character "a" is represented as 97 and "v" is represented as 118, and " \%" is represented as 37, so, for example the "av\%" converted to the number "1189737" but according to the number"1189737" we can't discover which message associated by ASCII even if we forwarded to dividing it's digits because we don't know the right mechanism for dividing!, not only for that, but also we want to represent the text as not big decimal numbers as possible as we can, to be able to apply RSA algorithm on that whole text directly in an efficient way.

In the following section we will illustrate our approach to be able to coming back to the original message and how we will use RSA algorithm with this approach. 
International Journal on Cryptography and Information Security (IJCIS), Vol. 5, No. 3/4, December 2015

\section{Proposed Solution And Associated Approach With RSA.}

We know that ASCII table[3] consists of 256 characters and the representation of them as numbers from 0 to 255 , so, suppose the group $\mathrm{A}$ is equal to $Z_{255}=\{0,1,2 \ldots \ldots 255\}$, and let's define the function $f$ as form: $f: A \rightarrow\{0,1\}^{8}$. It's easy to know the number of elements of $\{0,1\}^{8}$ is $2^{8}=256$. Indeed, $f$ is bijective function and so, it has inverse function. We can represent every dicimal number in $\mathrm{A}$ as a binary number by using binary and decimal number systems principles [5], and every number consists of 8 of 0 s and $1 \mathrm{~s}$.

(we can add some 0 s to the left of that representation if the number of digits is not 8 ), the same, we can represent every character as binary representation and every binary consists of 8 of 0 s and $1 \mathrm{~s}$.

Now, we will reivew our approch as algorithm, so suppose the message $M$ which we want to encrypt it, this algorithm illustrates the encryption role and get Cipher "C"(gibberish) as form:

1- Convert every character of $M$ to binary representation(every character must consists of 8 of $0 \mathrm{~s}$ and $1 \mathrm{~s})$ from left to right.

2- Convert whole representation(S) of $\mathrm{M}$ to decimal representation $M_{1}$.

3- Apply RSA encrypting function on the number $M_{1}$ and get number $M_{2}$.

4- Convert the number $M_{2}$ to binary representation(but the number of digits must divided by 8 ...we can do that by adding zeros to the left of representaion)

5- Divide that representation into bytes $(8 \mathrm{~s}$ of $0 \mathrm{~s}$ and $1 \mathrm{~s})$ from left to right.

6- Convert every byte in step 5 into asochiated character in ASCII from left to right and this creates cipher text $\mathrm{C}$.

To come back the original message "M", the following algorithm illustrates that:

1- Convert every character of $\mathrm{C}$ to binary representation(every character must consists of 8 of 0 s and 1s) from left to right.

2- Convert whole representation of $\mathrm{C}$ to decimal representation $M_{2}$.

3- Apply RSA decrypting function on the number $M_{2}$ and will get $M_{1}$.

4- Convert the number $M_{1}$ to binary representation(S)(but the number of digits must divided by 8 ...we can do that by adding zeros to the left of representaion)

5- Divide that representation into bytes( $8 \mathrm{~s}$ of $0 \mathrm{~s}$ and $1 \mathrm{~s}$ ) from left to right.

6- Convert every byte in step 5 into asochiated character in ASCII from left to right and this creates the original message text $\mathrm{M}$.

REMRK (1): we can stop at step(4)in encryption algorithm above and get cipher as a dicimal number, but in this case we will begin from step(4)in the decryption algorithm mentioned above.

REMRK (2): within applying this approch we have to be attantion in conditions of RSA algorithm wich related of message when represented as dicimal number because of the RSA not applicable if this number isn't less than the modulo $n(n=p * q)$ (see [2] ). 
International Journal on Cryptography and Information Security (IJCIS), Vol. 5, No. 3/4, December 2015

\section{NUMERICAL EXAMPLE.}

Suppose the message $\mathrm{M}$ as following:

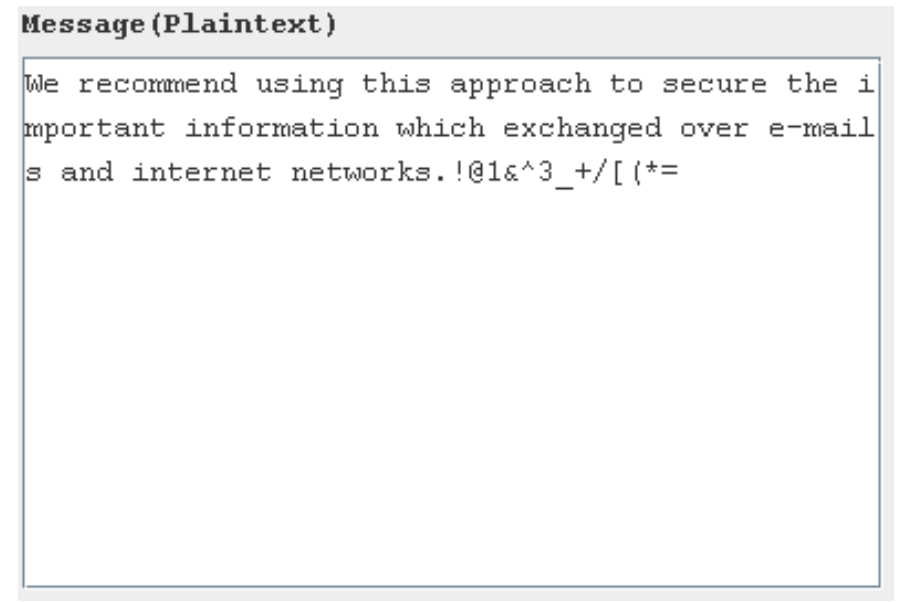

And we want to apply our approach with RSA, so, in the beginning let's build the RSA requirements $(p, q, n e, d, \emptyset(n))$ as mentioned above.

By using Miller-Rabin Test algorithm [2][4], we generated a 600-bits prime numbers $\mathrm{p}$ and $\mathrm{q}$ using java language[4][5] and get the following:

$\mathrm{p}=36816687633241002182065576654551879280165753023812270675138477824004277853846$ 1783870579961419016808201088329579401454411226957952898605246763846960198591381 6991755581819544236809351.

$\mathrm{q}=27186794025716014479926827223597630969441403917800649988487626523291402111446$ 8180061170641218156523178466332440217980586197709849237029353231432585315328291 0361359949026209979061959.

$\mathrm{n}=\mathrm{p} * \mathrm{q}=1000927703394049150956735889190158060942691067859650131675672227138305622$ 1323278974381732635552684572037879606487115336485429198732097926353032301768089 6095348004285611010290324917226394682382934162876830588655185205717499735657774 2468864641126802282282262244313854572630034076738036034391558203350478162209554 3676643245314918654260267696220413400258082099578609.

So, $\emptyset(n)=(p-1) *(q-1)=$ 1000927703394049150956735889190158060942691067859650131675672227138305622132327 8974381732635552684572037879606487115336485429198732097926353032301768089609534 8004285611010290324917162391200723977146214838184777035695467892578716161326222 8380083329325482609314349922822027396903406656479729538583915480737541752232029 7245362123199121070968867297869412327883707300.

Select number e $; 1<e<\emptyset(n)$, such that: $\operatorname{gcd}(e, \emptyset(n))=1$, let take $\mathrm{e}=11$; 
International Journal on Cryptography and Information Security (IJCIS), Vol. 5, No. 3/4, December 2015

And the number d(inverse product) will be :

$\mathrm{d}=45496713790638597770760722235916275497395048539075005985257828506286619187833$ 0862471896937979667480547176345749414333476610418124186269379683286444004073160 6727467527773195014768961926872760180779373401735671683440703086026305280060282 8562731060423885573150652269219183063495609393476351342662905249124433716010546 80566073692363236850440394226304278330856230591.

So, the public- key is (e,n) and private-key is $(\mathrm{d}, \mathrm{n})$.Now, let's apply our approach which mentioned above step by step, to encrypt message $\mathrm{M}$.

1- The binary representation of the message (character by character) is: $\mathrm{S}=$ 010101110110010100100000011100100110010101100011011011110110110101101101 011001010110111001100100001000000111010101110011011010010110111001100111 001000000111010001101000011010010111001100100000011000010111000001110000 011100100110111101100001011000110110100000100000011101000110111100100000 011100110110010101100011011101010111001001100101001000000111010001101000 011001010010000001101001011011010111000001101111011100100111010001100001 011011100111010000100000011010010110111001100110011011110111001001101101 011000010111010001101001011011110110111000100000011101110110100001101001 011000110110100000100000011001010111100001100011011010000110000101101110 011001110110010101100100001000000110111101110110011001010111001000100000 011001010010110101101101011000010110100101101100011100110010000001100001 011011100110010000100000011010010110111001110100011001010111001001101110 011001010111010000100000011011100110010101110100011101110110111101110010 011010110111001100101110001000010100000000110001001001100101111000110011 01011111001010110010111101011011001010000010101000111101.

2- The decimal representation of $\mathrm{S}$ is:

$M_{1}=674779901925981090940264347938001282716237591256227670169614914057960$ 882743836443699701318079498138580225881765380967217303146583780732812656 111452862620735498353002748845442215768238953985343249300352185989716276 564725635283466626213032688391167351175804072921362768408803847685904489 44662517840792486693738633291639357.

3- Applying RSA Encryption on $M_{1}$ and get $M_{2}$ as following:

$$
M_{2}=M_{1}^{e}(\bmod n)=
$$

863062826742439700286535333905757029720207366910955251723582169330998889 334187101465152792779967024367063523002136275498607043397142939850644957 851666561154751260571623481264893147279833000162802646942400233819241566 533597070669145406550404565169899133453077460458404471104791143022106721 525602611893089331489685531217659064283488939741765624469536510261947451 3.

4- binary representation of $M_{2}$ (with 0 s which added to the left) is:

100000000101000101101000011010001000100000000001100110101110110111010101 000011010110001011000000111110110100001110110100010100110100000000110011 110010001000110011010011011100111110000101110000100001110111000100000101 010111101101010111010110110110101100011000111111101110011101101001001110 011101011100110101110010010110010011011011001100111100110110011011110011 011011110100010000100010111101111111101100011111011000011001110000111011 101011111001011010101011110011001011110100101100001101001001000011101010 
International Journal on Cryptography and Information Security (IJCIS), Vol. 5, No. 3/4, December 2015

110111001100000101100010111110000001000111110111110001111011011001001011 111111110111101100100011000001110100011011111000110010001111000001000111 011001100011011110111011101101100111100011001000111100111110110010100011 111000010100100100101111010100000111111111100000110100011101111111010100 100010010111101100111101000000100101101011110001000011100000010101000001 010110011010100110001000001000110111110110100011111111110111010101011100 100001010100100011000111100011011111010101010111101101001110101111110001 001110111100010011101010100010011101100010011000000111110101000111010100 011001000111101100100100001001001100011000111101010011111101011010010110 010101001101100110111001001001100011101001010001.

5- Divide the representation in step (4) into 8s from "10000000" to the last byte which is"01010001"

6- Convert every byte from "10000000" to "01010001" into associated character in ASCII and get the cipher $\mathrm{C}$ (more gibberish )as form:

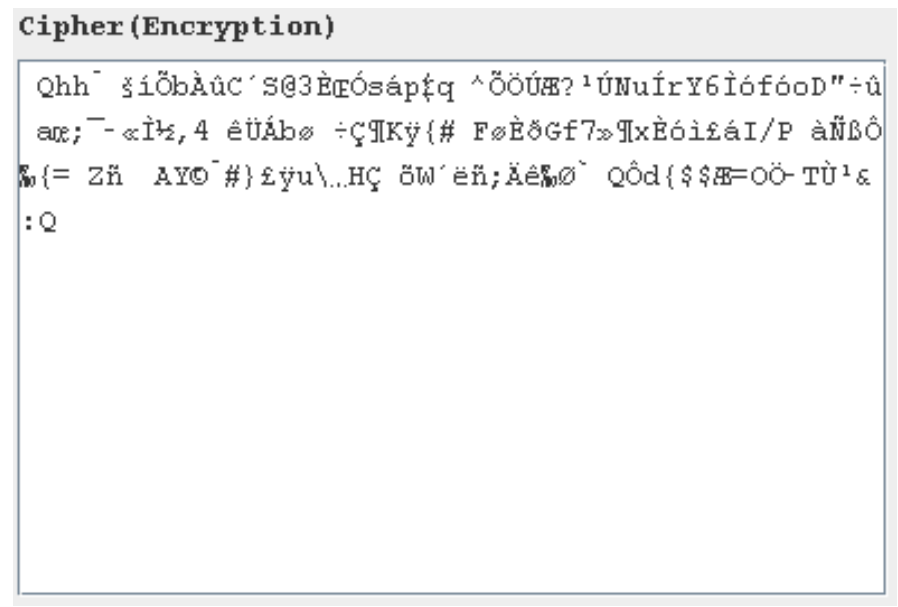

NoTE: in cipher above there are some characters disappear.

Now, suppose the receiver wants to do decryption to knows the original message $\mathrm{M}$ !, our decryption approach illustrates the mechanisem as the following:

1- The binary representation of the cipher $\mathrm{C}$ (character by character) is the same as in step (4)above in example(every representation of character must be byte): $\mathrm{L}=$

2- $10000000 \phi 1010001 \phi 1101000011010001000100000000001100110101110110111010101$ 000011010110001011000000111110110100001110110100010100110100000000110011 110010001000110011010011011100111110000101110000100001110111000100000101 010111101101010111010110110110101100011000111111101110011101101001001110 011101011100110101110010010110010011011011001100111100110110011011110011 011011110100010000100010111101111111101100011111011000011001110000111011 101011111001011010101011110011001011110100101100001101001001000011101010 110111001100000101100010111110000001000111110111110001111011011001001011 111111110111101100100011000001110100011011111000110010001111000001000111 011001100011011110111011101101100111100011001000111100111110110010100011 111000010100100100101111010100000111111111100000110100011101111111010100 100010010111101100111101000000100101101011110001000011100000010101000001 
International Journal on Cryptography and Information Security (IJCIS), Vol. 5, No. 3/4, December 2015

010110011010100110001000001000110111110110100011111111110111010101011100 100001010100100011000111100011011111010101010111101101001110101111110001 001110111100010011101010100010011101100010011000000111110101000111010100 011001000111101100100100001001001100011000111101010011111101011010010110 010101001101100110111001001001100011101001010001..

3- The dicimal representation of $\mathrm{L}$ is $M_{2}=$ 863062826742439700286535333905757029720207366910955251723582169330998889 3341871014651527927799670243670635230021362754986070433397142939850644957 851666561154751260571623481264893147279833000162802646942400233819241566 533597070669145406550404565169899133453077460458404471104791143022106721 525602611893089331489685531217659064283488939741765624469536510261947451 3.

4- Applying RSA Decryption on $M_{2}$ and get $M_{1}$ the same as in the step (2) in encryption algorithm above as following:

$M_{1}=M_{2}^{d}(\bmod n)=$ 674779901925981090940264347938001282716237591256227670169614914057960882 743836443699701318079498138580225881765380967217303146583780732812656111 452862620735498353002748845442215768238953985343249300352185989716276564 725635283466626213032688391167351175804072921362768408803847685904489446 62517840792486693738633291639357.

5- Convert $M_{1}$ into binary representation(adding zeros to the left of representation to be the length divided by 8 ) and we will get $\mathrm{S}$ the same in the first step in encryption algorithm above as following: $\mathrm{S}=$ 010101110110010100100000011100100110010101100011011011110110110101101101 011001010110111001100100001000000111010101110011011010010110111001100111 001000000111010001101000011010010111001100100000011000010111000001110000 011100100110111101100001011000110110100000100000011101000110111100100000 011100110110010101100011011101010111001001100101001000000111010001101000 011001010010000001101001011011010111000001101111011100100111010001100001 011011100111010000100000011010010110111001100110011011110111001001101101 011000010111010001101001011011110110111000100000011101110110100001101001 011000110110100000100000011001010111100001100011011010000110000101101110 011001110110010101100100001000000110111101110110011001010111001000100000 011001010010110101101101011000010110100101101100011100110010000001100001 011011100110010000100000011010010110111001110100011001010111001001101110 011001010111010000100000011011100110010101110100011101110110111101110010 011010110111001100101110001000010100000000110001001001100101111000110011 01011111001010110010111101011011001010000010101000111101.

6- Divide the last representation into bytes and you able to do that because the number of digits is divided by 8 .

7- Convert from left to right every byte in the last representation into associated character in ASCII and will be get the exactly message "M" : 
International Journal on Cryptography and Information Security (IJCIS), Vol. 5, No. 3/4, December 2015

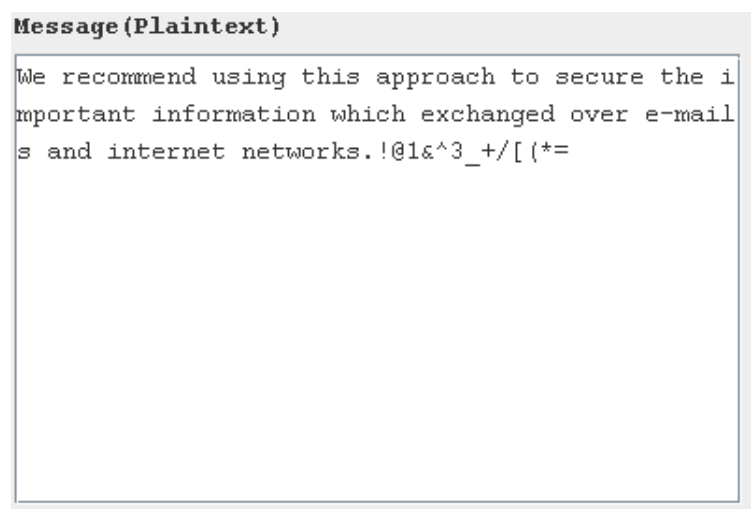

NOTE: We can see that decryption approach is absolutely true and will lead to the exactly original message, because it was built from last step to first step in the proposed encryption approach in this paper(it is the inverse of encryption approach step by step!).

\section{Java ApPlication For Proposed APPRoACH}

Depending on java language[4][5] we build an application to implimination our approach. In this application we can generate d1,d2-bits primes numbers $\mathrm{p}$ and $\mathrm{q}$ depending on Miller-Rabin Test algorithm[4][2], and we can directly apply RSA algorithm with our approach to encrypt and decrypt message text according to ASCII. Also, this application can tell us directly if the RSA algorithm is applicable with this approach or not(just when we put the message text wich we want incrypt it).

Figure(1) reviews the results of the numerical example mentioned above by our application, and the figure(2) appeares when the "go to decrypt the message" button is preesed to come back the original message according to the private key "d":

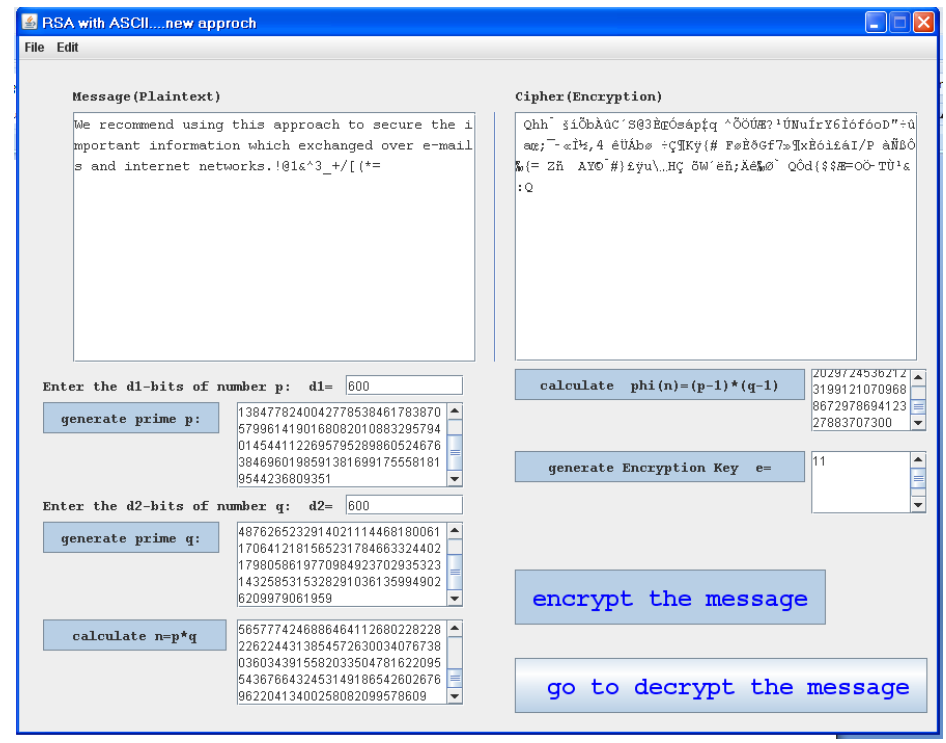

Figure(1): java application for encryption approach deppending on RSA and ASCII 


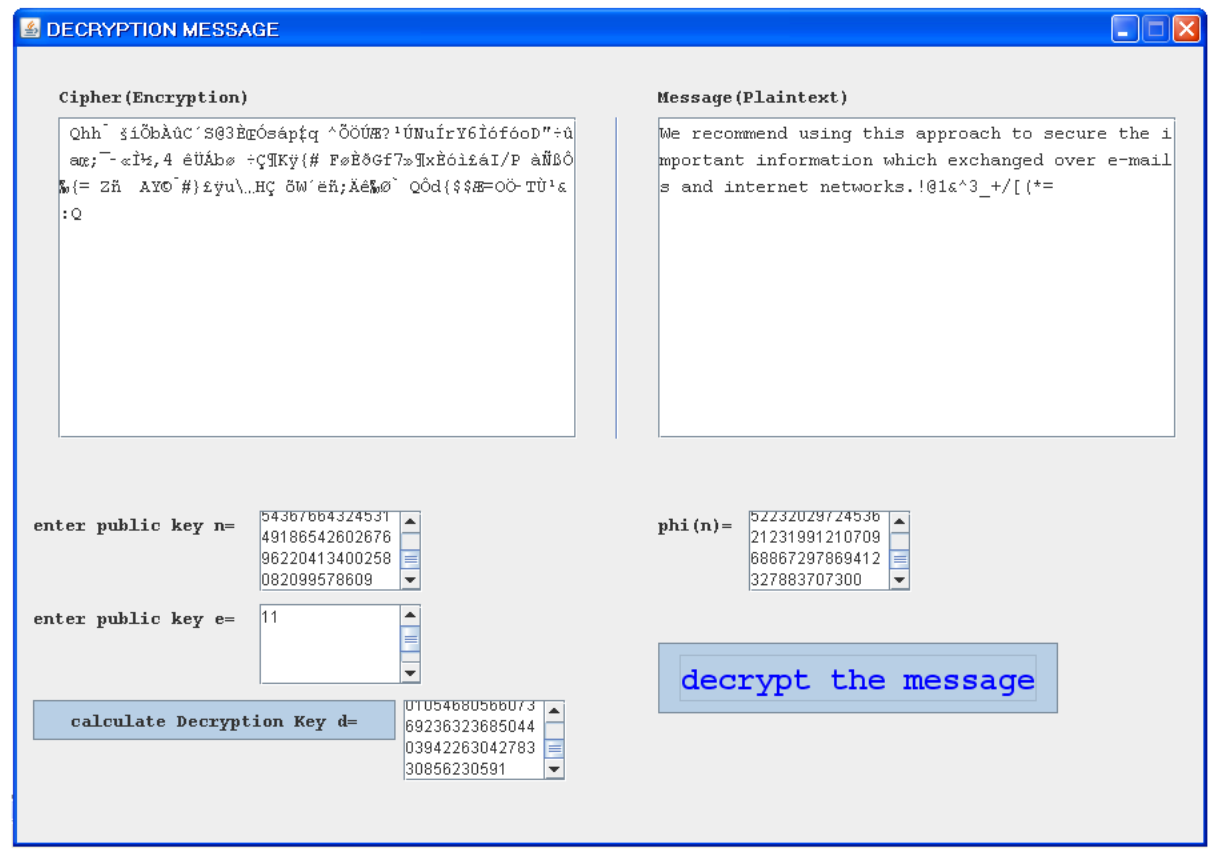

Figure(2): java application for decryption approach deppending on RSA and ASCII

\section{Conclusion And Future Plan}

This paper describes a new approach for encryption and decryption message text using RSA algorithm depending on ASCII, and java application to implementation this approach. We recommend using this approach to secure the important information which exchanged over e-mails and internet networks, even, secure the gibberish passwords.

Since the RSA algorithm will be slow when the message text is big, so, we recommend to use this approach after dividing the message into blocks and apply this approach on the every block in fast way while transmitting in the networks ...block by block, so we can build java applet to do that and this is will be our future plan with what the relationship between the modulo " $\mathrm{n}$ " in RSA and the number representation of the message to allow us dividing into blocks automatically in an efficient way.

\section{REFERENCES}

[1] William, Stalling,(2014), "Cryptography and Network Security-Principles and Practice", Sixth Edition, Pearson Education, Inc

[2] Song, Y. Yan,(2013), "computational number theory and modern cryptography“, 1st, Wiley.

[3] website Link: http://www.asciitable.com/ . On Thu Dec 24 20:38:46, 2015.

[4] David, Bishop, (2003), "Introduction to Cryptography with Java Applets",Jones and Bartlett Publisher.

[5] Y. Daniel Liang,(2013), "Introduction to Java Programming “ Ninth Edition, Pearson Education.. 
International Journal on Cryptography and Information Security (IJCIS), Vol. 5, No. 3/4, December 2015

\section{AUTHORS}

Ahmad Steef is a PhD Candidate in mathematics at Al-Baath University, Homs, Syria.

Mohammad Nour Shamma is a professor in mathematics at Damascus University- Basic Sciences departmentthe Mechanical and Electrical Engineering- Syria. His Research interests include mathematical analysis, Cryptography.

Abdulbaset Alkhatib is a professor in mathematics at Al-Baath University- Homs, Syria. His Research interests include Linear algebra, Cryptography. 